predictive genetics. However, no mention is made of the science of behavioural and cognitive phenotypes of genetically determined syndromes. Oddly ignored or unrecognised, this none the less important patient population is of direct concern to the child psychiatrist as well as to the adolescent and adult psychiatrist.

Since the early 1990s a number of syndromes known in clinical genetics have found a molecular explanation. To cite but a few (OMIM, 1996), the genetic alterations discovered and understood include those responsible for syndromes such as fragile $X$ (incidence 1 per 2000 children), VeloCardioFacial/DiGeorge/CATCH $22 \quad(0.5-2.5$ per 10000), Prader-Willi (1 per 25000), Angelmann (1 per 25000) and William's (1 per 20000 ). All these syndromes, the physical phenotype (dysmorphism) of which may be inconspicuous, also express a characteristic psychiatric phenotype. Presently, it is possible to establish genotype-psychiatric phenotype correlations. At the psychiatric level such investigations lead to knowledge which, in turn, allows for a more targeted guidance or psychoeducational patient approach. Furthermore, in a medium-term perspective, such research enables the development of study models for future investigations of cohorts of children carrying susceptibility genes, notably for schizophrenia, bipolar disorder or obsessivecompulsive disorder.

FARMER, A. \& OWEN, M. J. (1996) Genomics: the next psychiatric revolution? British Journal of Psychiatry, 169, 135-138. OMmM DATABase (1996) http://www3.ncbi.nlm.nih.gov/omim/

S. ElIEZ

Institute of Psychiatry

University of Geneva

Geneva 1206, Switzerland

\section{Depression and the safety of antidepressants}

SIR: The Defeat Depression Campaign might be expected to improve the detection of depression. However, if as a consequence potentially suicidal patients receive older, potentially more toxic tricyclic drugs rather than newer, safer antidepressants, then the risk of life-threatening or fatal overdose may reduce the likelihood of a successful treatment outcome.

Basic details of all deliberate self-harm (DSH) episodes reporting to Derby Royal Infirmary Casualty Department have been recorded on a database from 1990. A retrospective, case-note survey was conducted of deliberate antidepressant overdoses registered at the same department from 1 January 1995 to 31 December 1995. There were 179 self-poisonings with either an older tricyclic, a selective serotonin reuptake inhibitor (SSRI), or a 'novel', non-SSRI (either trazodone or lofepramine). Lofepramine or trazodone were equally likely to be used by those with or without a history of DSH (13 and 12 episodes, respectively). There was no significant differential association between overdose by older tricyclic or SSRI and a record of previous DSH (49 tricyclic and 41 SSRI overdoses in those without a history of DSH; 30 tricyclic and 34 SSRI overdoses in those with such a history, $\chi^{2}=0.858, P>0.1$ ). Of course, some overdoses may have involved medication not currently prescribed to the patient.

There appears to be considerable scope to promote the prescribing of the newer, less toxic antidepressants among those at risk of repeated self-poisoning, if depression is to be treated effectively but without undue risk.

Department of Health Care for the Elderly

S. THACKER Medical School, University Hospital

Nottingham NG7 $2 \mathrm{UH}$

\section{Incidence of mental disorder}

SIR: When reporting on the incidence of psychiatric disorders in Finland, Lehtinen et al (1966) indicate that information from other studies is limited and refer for comparison only to the Lundby Study in Sweden (Hagnell et al, 1990) and the Epidemiologic Catchment Area (ECA) Program in the USA (Eaton et al, 1989). Findings from at least two other studies would have filled out the picture of available evidence and strengthened their conclusions. One of these is a study of women in Scotland (Surtees et al, 1986). The other is an investigation my colleagues and I have carried out in Atlantic Canada, the Stirling County Study (Murphy et al, 1988).

Lehtinen et al (1996) conclude that "there are huge differences" in results about incidence. Average annual incidence of all types of mental disorders grouped together was 15 per 1000 in the 16-year Finnish study, 18 per 1000 in the 25-year Swedish study, but nearly 100 per 1000 in the one-year USA study.

Depression is used in the following to illustrate similarities and differences when the Canadian and Scottish studies are also brought to attention. The Stirling County Study is similar to the Finnish study in the size of the population investigated and the use of a 16-year follow-up period. Average 
annual incidence of depression in the Canadian study was 2.1 per 1000 for men and 2.5 per 1000 for women. This compared with 2.0 and 5.0 per 1000 in men and 2.7 and 7.0 per 1000 in women in Finland and in Sweden (the Lundby Study), respectively. The ECA study reported an incidence of DSM-III major depression of 11 and 20 per 1000 for men and women, respectively, and the Scottish study found incidence to be 78 per 1000 among women for RDC major depression.

Differences of this magnitude are troubling. I agree with the Finnish investigators when they say that these differences may be influenced by diagnostic criteria and the length of the follow-up period. To these reasons I would add that different styles of interviewing and variant forms of questions may be implicated. Nevertheless, I suggest that the rates from Canada, Finland and Sweden are more reasonable than the higher rates, because they fit better with evidence that psychiatric disorders are often chronic or recurrent and that, under such conditions, incidence is typically low relative to prevalence.

The most compelling reason, however, for doubting the credibility of the higher rates relates to their long-term implications. Suppose that the Scottish and USA rates pertain to a group of women aged 20 who are at risk for a first-ever major depression. By the time they are 35 years old, virtually all of the Scottish cohort would have succumbed to this disorder. Using the not-quite-so high data from the USA, if all women in such a cohort survived to age 70 , all would have experienced at least one episode of major depression. If everybody at some time has a 'major depression', is major depression the serious disorder that clinical evidence indicates it is?

Eaton, W. W., Kramer, M., Anthony, J. C., et al (1989) The incidence of specific DIS/DSM-III mental disorders: data from the NIMH Epidemiologic Catchment Area Program. Acta Psychiatrica Scandinavica, 79, 163-178.

HaGnell, O., Essen-Möller, E., LANKe, J., et al (1990) The incidence of mental illness over a quarter of a century. Stockholm, Sweden: Almquist \& Wiksell.

LeHTnNen, V., VeuOLA, J., Lndholx, T., et al (1996) Incidence of mental disorders in the Finnish UKKI Study. British Journal of Psychiatry, 168, 672-678.

MurPhy, J. M., Ouvier, D. C., Monson, R. R., et al (1988) Incidence of depression and anxiety: the Stirling County Study. American Journal of Public Health, 78, 534-540.

Surtees, P. G., Sashimharan, S. P. \& Dean, C. (1986) Affective disorder amongst women in the general population: a longitudinal study. British Journal of Psychiatry, 148, 176-186.

Department of Psychiatry

J. M. MURPHY

Harvard Medical School

Boston, MA 02114-2698

\section{Women and psychiatry}

SIR: Crimlisk \& Welch (1996) discuss interesting data concerning women as psychiatrists and gender differences in aptitude for psychiatry among medical students. The latter topic has also been recently reviewed by Eagles (1996). However, their interpretation of the Royal College of Psychiatrists census material may not be entirely correct. The absence of a gradient in the proportion of women in the various training grades does not rule out a cohort effect as an explanation of the relative dearth of female consultants. A breakdown of the proportion of women in the consultant grade at various seniority levels would be needed to prove or disprove this. Unfortunately, the census does not yet obtain this detail but some information from other sources indicates that women are relatively over-represented in the earlier years of consultant status. Ideally, prospective studies of the current trainee cohort would be needed to assess the relative success rate of men and women in moving to the consultant grade.

This does not detract from the value of the suggestions made by Crimlisk \& Welch and by Eagles that flexibility of working patterns should be maximised in the training grades and in career posts to enable all doctors, particularly those with domestic commitments, to pursue their chosen career path. Encouragingly, recent meetings between representatives of the College and the Department of Health have established a considerable measure of agreement on the desirability of such flexibility and a commitment towards finding methods of achieving it.

Crnalsk, H. \& Welch, S. (1996) Women and psychiatry. British Journal of Psychiatry, 169, 6-9.

EAGles, J. M. (1996) Gender differences in attitudes and recruitment into psychiatry. Psychological Reports, 78, 653-654.

\section{The General Infirmary at Leeds}

D. STORER

\section{Great George Street}

Leeds LS1 3EX

SIR: We read with interest the editorial by Crimlisk \& Welch (1996). We wish to broaden the scope of their review by focusing firstly, on the influences of gender on psychotropic drug use and secondly, on drug metabolism. Yonkers et al (1992) have reviewed the theoretical background and the evidence supporting gender-related differences in pharmacokinetic and pharmacodynamic properties of psychotropic medication. Their findings suggest that young women seem to respond better to and 